\title{
Classification of African Mosaic Virus Infected Cassava Leaves by the Use of Multi-Spectral Imaging
}

\author{
Mama Sangare1, Thérèse Atcham Agneroh'2, Olivier Kossan Bagui ${ }^{3}$, Issiaka Traore1, \\ Abdramane Ba ${ }^{1}$, Jeremie Thouakesseh Zoueu $^{3^{*}}$ \\ ${ }^{1}$ Laboratoire d'Optique, de Spectroscopie et des Sciences de l'Atmosphere, Université des Sciences, \\ Techniques et Technologies de Bamako (USTTB), Bamako, Mali \\ ${ }^{2}$ Laboratoire de Phytopathologie et Biologie Végétale, Institut National Polytechnique Felix \\ Houphouët-Boigny, Yamoussoukro, Cote d'Ivoire \\ ${ }^{3}$ Laboratoire d'Instrumentation Image et Spectroscopie, Institut National Polytechnique Felix \\ Houphouët-Boigny, Yamoussoukro, Cote d'Ivoire \\ Email: ${ }^{*}$ jeremie.zoueu@inphb.edu.ci
}

Received 6 August 2015; accepted 28 August 2015; published 31 August 2015

Copyright (C) 2015 by authors and Scientific Research Publishing Inc.

This work is licensed under the Creative Commons Attribution International License (CC BY). http://creativecommons.org/licenses/by/4.0/

\begin{abstract}
In this work, we have used multispectral imaging technology to classify cassava leaves infected by African mosaic virus by the use of their unique spectral finger print. The spectra are extracted from transmission, reflection and diffusion of their multispectral images; they have been then analyzed with statistical multivariate analysis techniques. Principal component analysis (PCA) has been used followed by K-means and Ascending Hierarchical Classification (AHC) to endorse the classification. The contribution of this work is the use of multispectral imagery which binds both spatial and spectral information to differentiate and sort infected leaves. The results show that the multimodal and imaging spectroscopy may allow blind identification and characterization of infected leaves.
\end{abstract}

Keywords

Classification, Infected Leaves, African Cassava Mosaic Virus, Multispectral Imaging

\section{Introduction}

Cassava is a food crop in the humid tropical zone. Ivory Coast produces about 1.7 million tons with yields rang-

"Corresponding author.

How to cite this paper: Sangare, M., Agneroh, T.A., Bagui, O.K., Traore, I., Ba, A. and Zoueu, J.T. (2015) Classification of African Mosaic Virus Infected Cassava Leaves by the Use of Multi-Spectral Imaging. Optics and Photonics Journal, 5, $261-272$. http://dx.doi.org/10.4236/opj.2015.58025 
ing between 5 and 8 tons per hectare [1]. Every year in Ivory Coast, some major factors reducing the production of cassava are bacterial, viral and fungal diseases and nematodes [2]. Among the diseases, African cassava mosaic induces important yield losses in cassava. The symptom due to African cassava mosaic virus (ACMV) is leaf deformation, leaf narrowing and stunting of the plant [3]. African cassava mosaic virus has been studied by many researchers. In 1994, BCA hohuendo and S. Sarkarmadeanultra-structural study of plant cells was infected by the virus using the method of electron microscopy to characterize the cellular components of healthy and infected leaf tissues [4]. A study conducted in 2009 also focused on a statistical approach for visual diagnostic of symptoms of ACMV on plant leaves in order to show viral resistance by grafting [5]. Other researchers made a classification of species of cassava plants in the 1970's [6]. The study of the transmission of ACMV is not observed by light microscopy and electron microscopy [7]. In Africa namely in Ivory Coast, no work is done on the multi-spectral imaging for spectral characterization of symptoms caused by ACMV. Multispectral microscopy is an expanding area and its applications range from medical diagnostics to the environment passing by agriculture. It is a coupling between optical spectroscopy and microscopy. This method determines a correlation between the spatial and spectral information resulting from the light-matter interaction. Indeed, for a given wavelength, we obtain a multivariate image resulting from two spatial dimensions that determine a point or pixel of the image and a spectral dimension which is the spectrum associated to the pixel.

We propose in this work a study based on multi-spectral imaging to characterize some symptoms caused by ACMV.

\section{Materials and Methods}

\subsection{Biological Sample}

Samples were collected during a prospection in a cassava field in the north of the National Polytechnic Institute Felix Houphouet-Boigny (INP-HB) Yamoussoukro/Ivory Coast. All the leaves were practically infected by the African cassava mosaic. We selected three leaves of cassava plants:

- A healthy leaf as a reference;

- An infected leaf in which the limb has been deformed;

- An infected leaf in which there has been a color change on its interface.

\subsection{Methods of Data Acquisition}

The data acquisition system has been described in [8] [9]. The classical luminous sources have been removed and replaced for all modes, by a set of 13 LEDs, ranging from $375 \mathrm{~nm}$ to $940 \mathrm{~nm}$. This enables the spectral coverage of this region with a mean step of $40 \mathrm{~nm}$. The initial mechanical oculars are substituted by a monochrome camera CMOS 12-bit (2592 × 1944, Guppy-503B, Vision Allied Technology, with a sensor of MT9P031 micron/Aptina), with a pixel size of $2.2 \mu \mathrm{m} \times 2.2 \mu \mathrm{m}$, which is used to acquire images. The system acquires automatically a total of 39 spectral images; 13 images by mode), for the same scene using a data acquisition card (NI-DAQ) coupled to a computer, which controls the intensities of current for fine adjustments. In this work, we visualized the leaves naturally infected by the African cassava mosaic with three modes, transmission, reflection and scattering, because they better reveal the presence of substances in the cell. The thirteen luminous sources are sufficient to study the optical properties of biological cells [10]. We get images of leaves infected by ACMV and their spectra of transmission, reflection and scattering (see results below).

\subsection{Data Analysis}

The images that we treated are of three dimensions, characterized by two spaces: the image space that accounts on the spatial dimension and spectral space representing the spectral dimension. The regions of interest correspond to the images of cassava leaves. In these regions, the objects, which are analyzed and should be characterized, are the naturally stressed leaves by ACMV. In image space, each of those objects is characterized by a repartition of intensities of the pixels representing them; objects are observed following 13 channels. If $N$ is the number of channels corresponding to spectral bands, each pixel observed along the $N$ channels will be represented by a vector $X$ belonging to a sub vector space of dimension $N$. The objective of this work requires that the $X$ vectors are identified and grouped into $M$ classes corresponding to different symptoms caused by ACMV present in the leaf. The data analysis method can be summarized by the flow chart below (see Figure 1). 


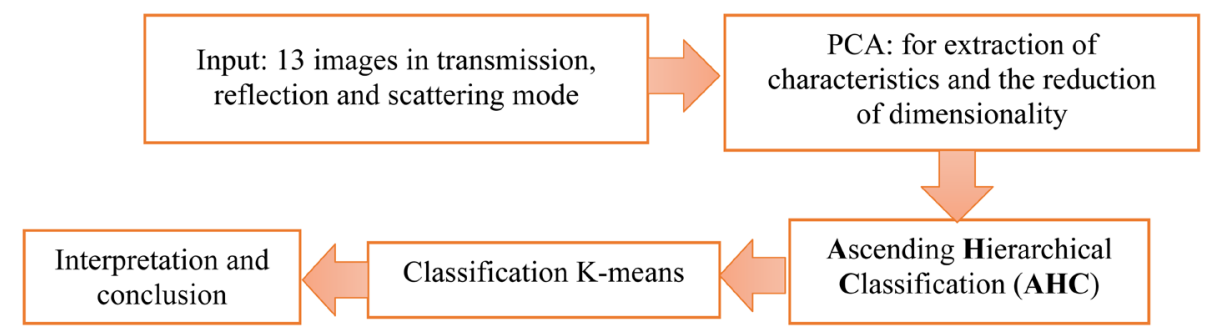

Figure 1. Data analysis process.

\section{Results and Discussion}

\subsection{Results}

We have images of 75 cassava leaves (25 healthy leaves, 25 leaves with deformed limb, 25 light-green and dark green leaves). The microscopic image shows a difference in transmission mode, reflection and scattering (see Figure 2).

The spectral signatures of healthy and infected cassava leaves by the African cassava mosaic in the three modes have redundancies of information (Figure 3). To reduce this redundancy by keeping the maximum of information, we will apply the method of principal component analysis [11]. By applying PCA we have condensed the data for each vector $X$ into new groups so that they don't present any correlation between them and are ordered in terms of the percentage of variance brought by each component. Thus, the first principal component contains information related to the maximum variance, the second contains information related to the following variance. The process is repeated up to the $\mathrm{n}^{\text {th }}$ principal component. The loss of information decreases from one stage to the next. So we obtain the principal component $Y_{i}$ with $i=1, \cdots, I$ and $I \leq 13^{\circ}$. We have:

$$
Y_{i}=\sum_{j=i}^{N}\left[V_{j}\left(X_{i}^{j}-\bar{X}_{i}\right)\right]
$$

These averages are used to calculate covariance and correlations between variables $X_{i}$ and $X_{i}(i=j)$.

$V_{j}$ : Represents proper vectors associated with proper values calculated from the polynomial of the matrix of covariance $S$ :

$$
S=\left\{\operatorname{Cor}\left(X_{i}, X_{j}\right)\right\}_{13 \times 13}
$$

However, the quality of the estimates, which led PCA, depends on the choice of the number of components retained to reconstruct the data, or the dimension of the subspace representation. So we have from the Eboulis criterion [12], which is a graph presenting the decreasing of the proper values reducing the number of dimensionality. The principal consists to search for if there is an "elbow" in the graph for each mode and keep only the proper values up to this elbow (Figure 4) for the selection of principal components. According to the Eboulis graph:

- Components 1and 2 can better describe our data in transmission and scattering mode;

- The components 2 and 3 also better describe our data in reflection mode;

- The projection of transmission, reflection and scattering mode in two dimensional pace of these principal components retained gives us the graphs in Figure 5.

Two or three classes are formed according to these projections in the three geometries. Healthy cassava leaves form a class, but the two leaves naturally infected by the African cassava mosaic seem to form a class together or two classes if the infected leaves are considered in the same class.

The results of our principal component analysis are difficult to interpret physically because certain points have a great dispersion compared to elements of their class [13]. So we made recourse to two cluster analysis methods: Ascending Hierarchical Classification and K-means classification. Both methods were applied to the data after the PCA. Graphs of Figure 6 gave the results after application of the Ascending Hierarchical Classification (AHC) and the K-means method.

The result of this classification method we give three distinct elements in transmission, reflection and scattering namely blue (healthy leaf), green (limb of a deformed leaf), red (bright-green and dark-green leaf). We have proposed another method (Ascending Hierarchical Classification or AHC) to verify the performances of K-means. After applying AHC, we have: 


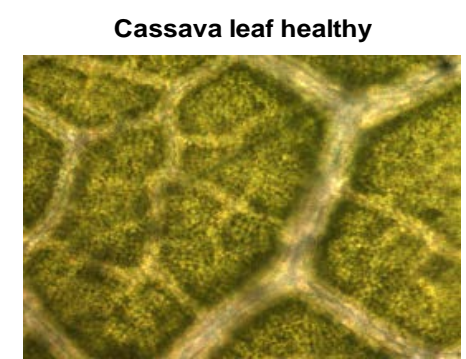

(a1)

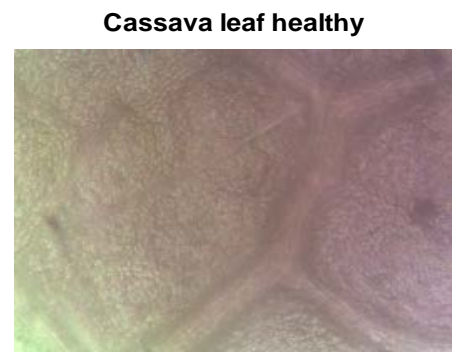

(b1)

Cassava leaf healthy

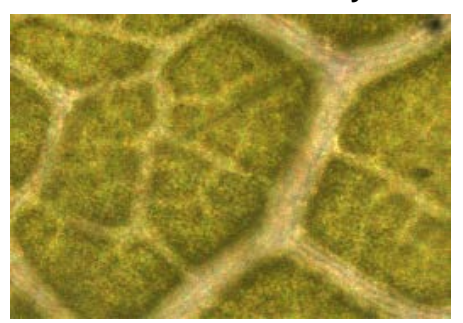

(c1)

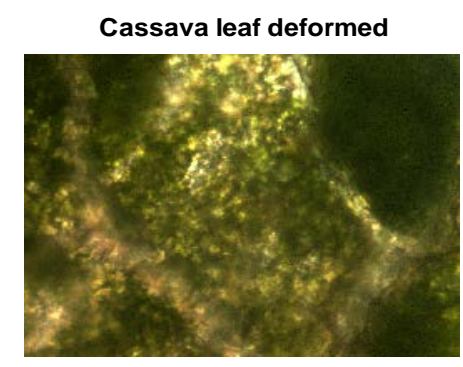

(a2)

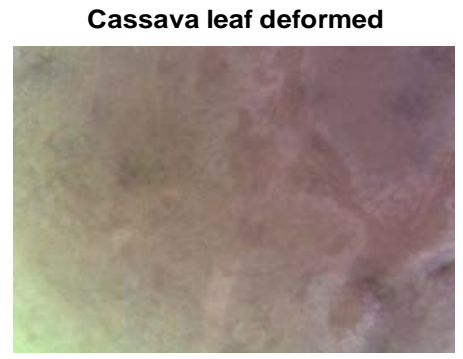

(b2)

Cassava leaf deformed

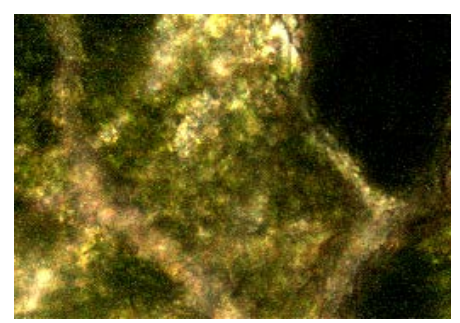

(c2)
Cassava leaf pale-green

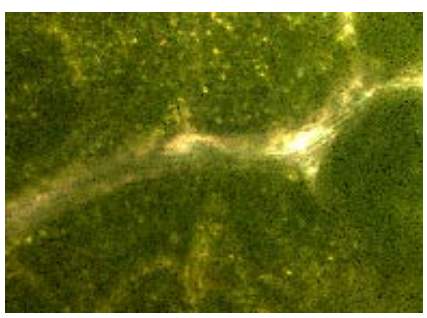

(a3)

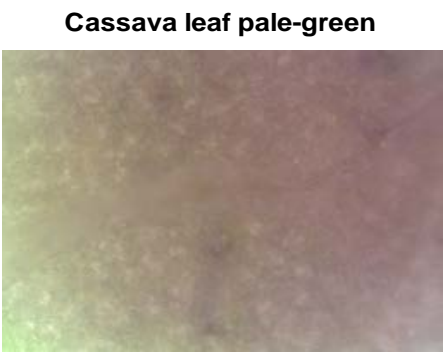

(b3)

Cassava leaf pale-green

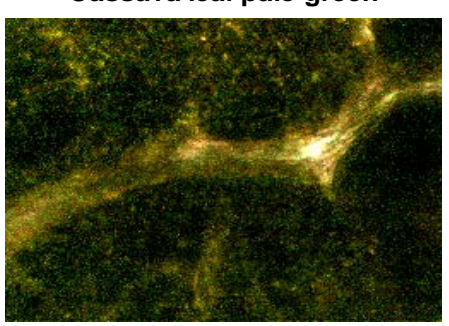

(c3)

Figure 2. Color sample images of cassava healthy (a1, b1, and c1), deformed (a2, b2, and c2), and pale-green (a3, b3, and c3) leaves, in transmission (a1, a2, and a3), reflection (b1, b2, and b3) and scattering (c1, c2, and c3) modes.

- A class in transmission mode;

- Three distinct classes in reflection mode;

- Two classes in diffusion mode (see the representation graphs in Figure 7).

The objective to verify the spectral characterization of our samples using the hierarchical cluster method was not attained. The collected samples were distinguishable with the naked eye. We just wanted to check that with a statistical approach to study their photosynthetic effect with the technique of optical spectroscopy. We can say that the K-means method is an ideal technique in our study. Finally, we get three distinct classes (graphs in Figure 8) after the analysis of the optical spectra means.

\subsection{Discussion}

\subsubsection{In Transmission Mode}

The healthy cassava leaf (class 1), has a light transmission variation in terms of intensities from ultraviolet to near infrared according to thirteen wavelengths used. Such behavior is explained by the fact that the healthy leaf is formed of exposed chloroplasts to daylight; and visually, it was not green. Thus, the healthy cassava leaf can't resist the attack by the African cassava mosaic because it doesn't normally play the role of photosynthesis. Its rate of chlorophyll concentration varies from one wavelength to another. At this point, during the young age, the healthy leaf has a high vulnerability to African cassava mosaic. Despite this variation of chlorophyll pigments, a cassava plant formed of healthy leaves can give a good yield. However, in the same bands used in transmission mode, the two infected leaves by the African cassava mosaic that we have studied similar spectral signature. The spectrum of the limb of a cassava leaf deformed (class 2) by this virus has a higher level of transmission in terms 


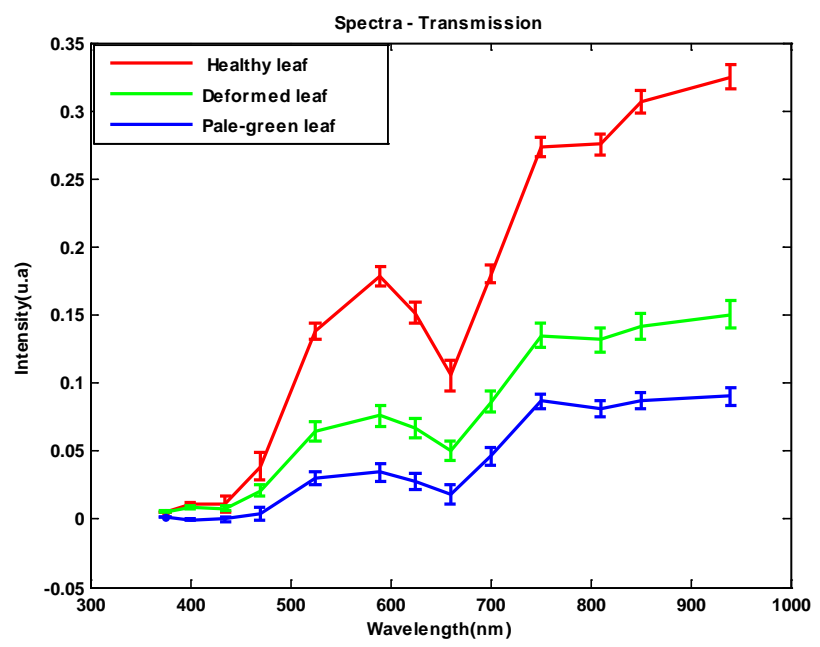

(a)

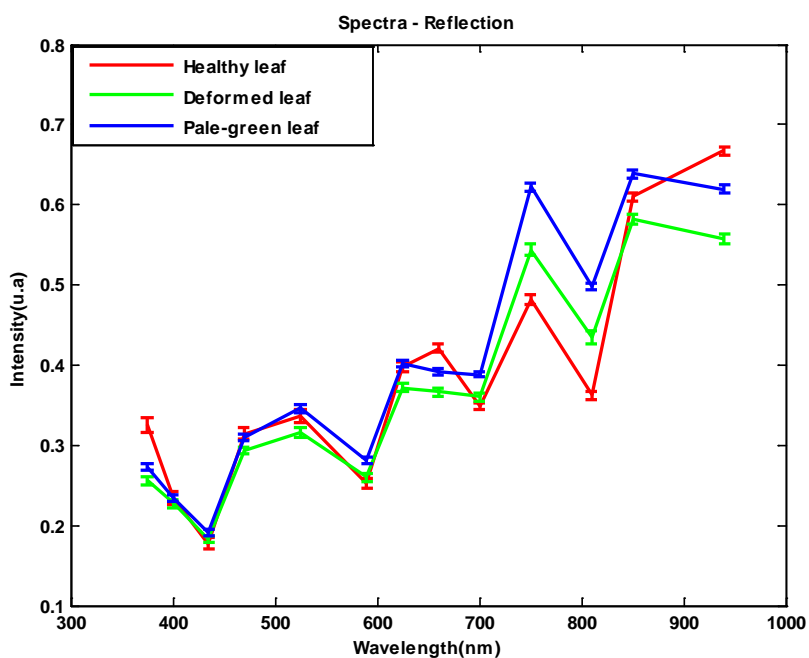

(b)

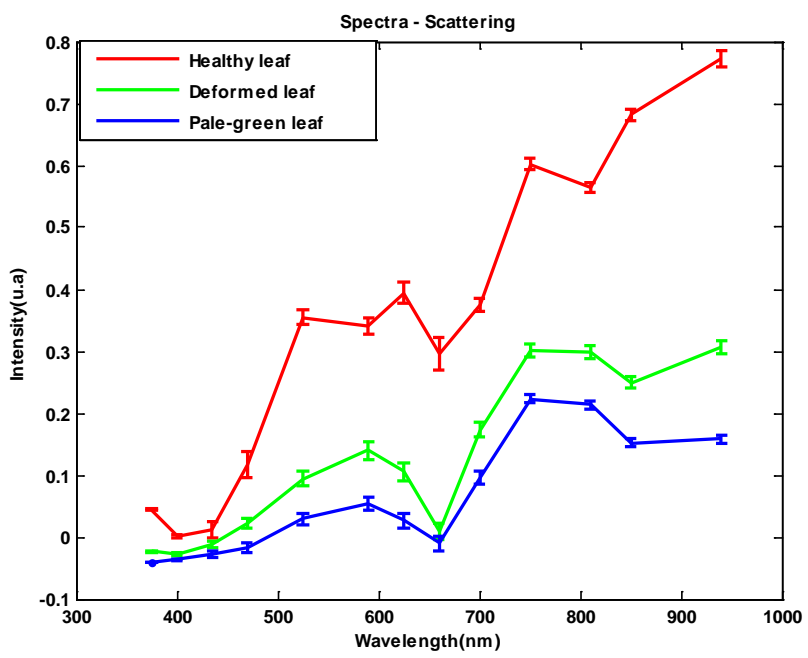

(c)

Figure 3. Comparison of healthy, deformed and pale-green leaves spectra in transmission (a), reflection (b) and scattering (c) modes. 


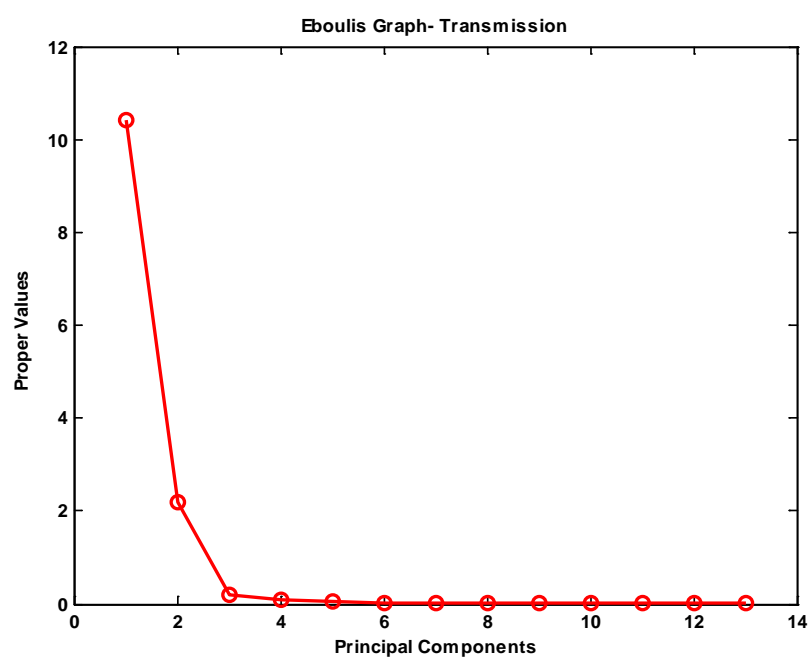

(a)

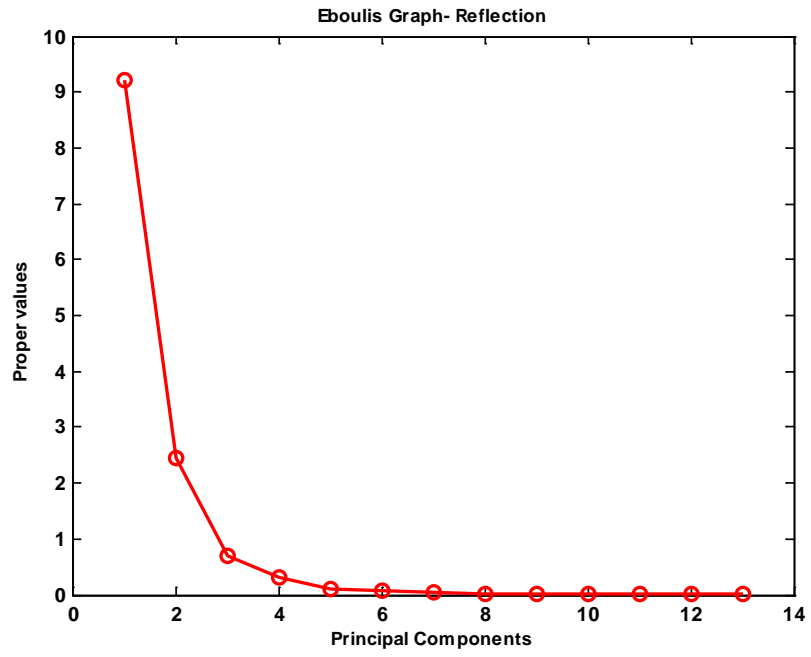

(b)

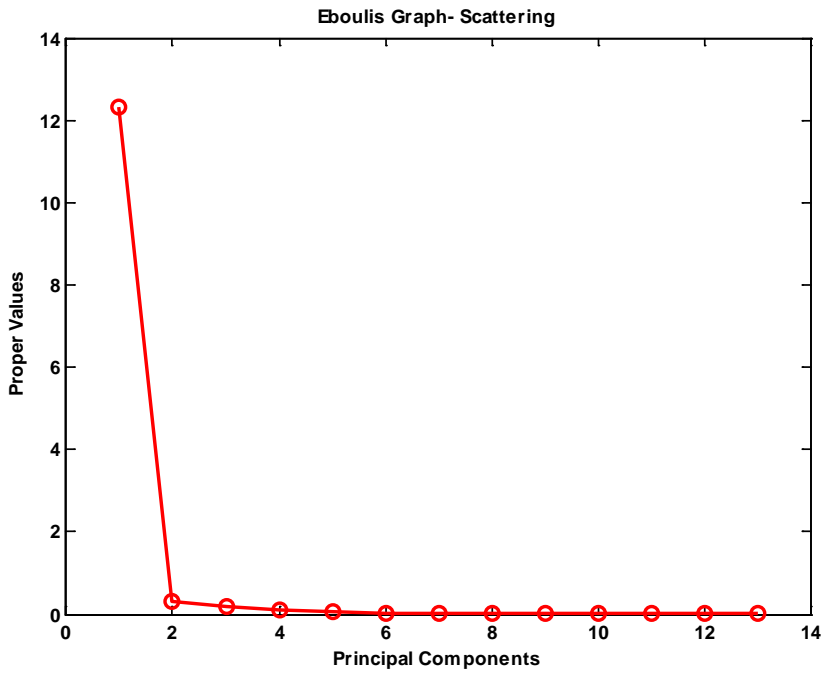

(c)

Figure 4. Comparative Eboulis representation of cassava leaves in transmission (a), reflection (b) and scattering (c) modes. 


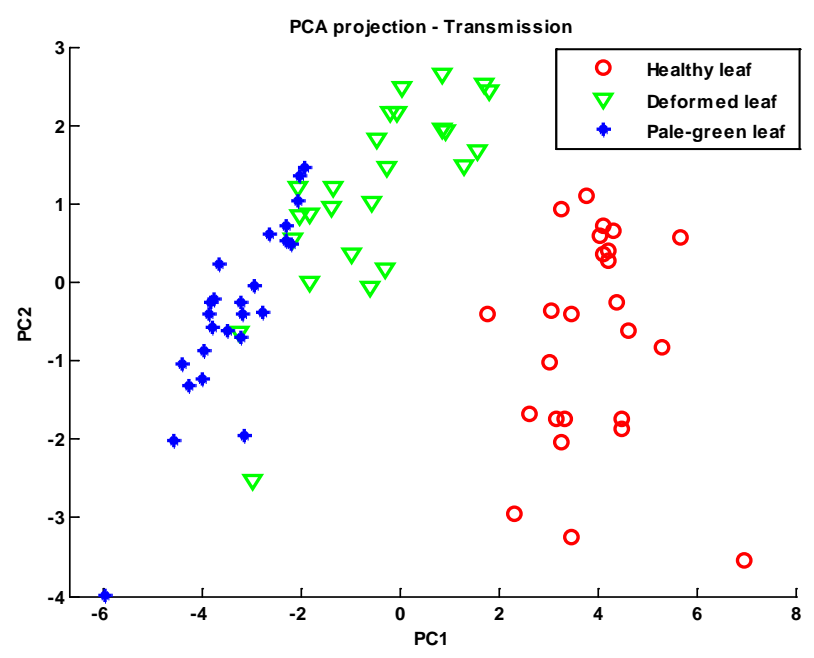

(a)

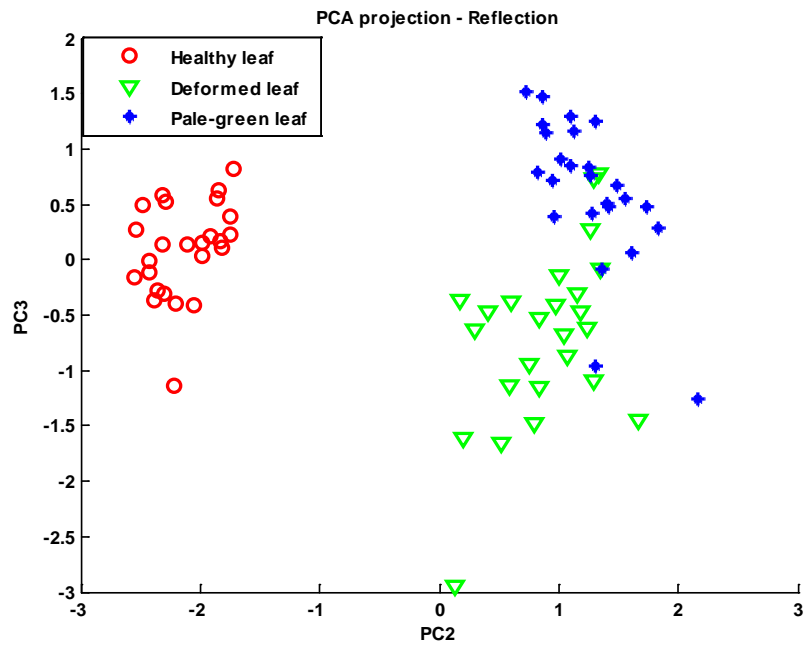

(b)

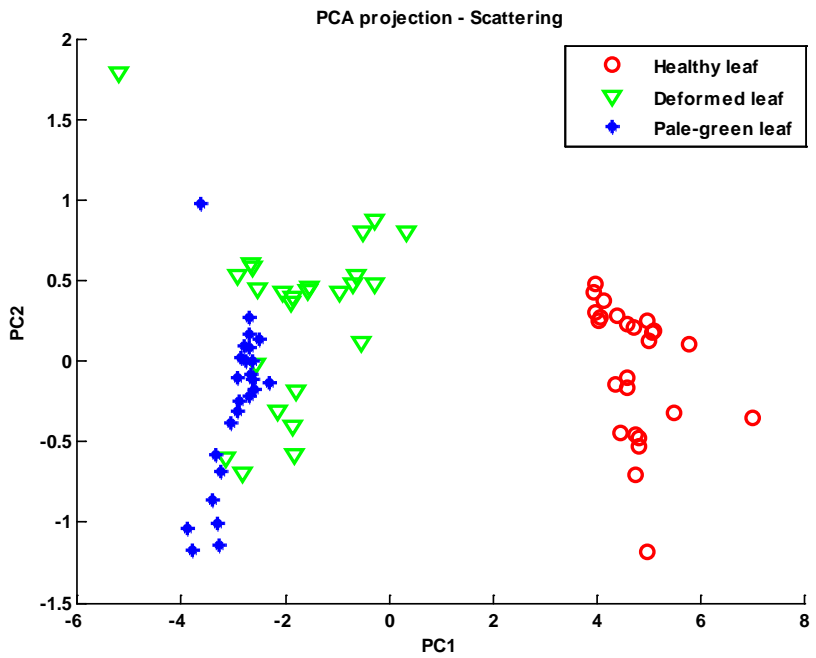

(c)

Figure 5. Comparative projection of cassava leaves infected by the African cassava mosaic virus in transmission (a) reflection (b) and scattering (c) modes, using PC1 and PC2 mode using PC1 and PC2. 


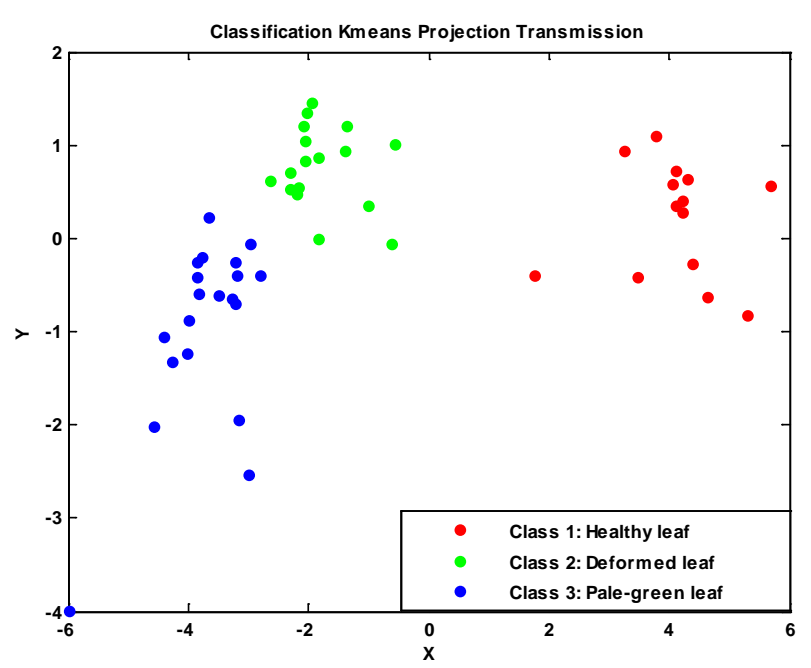

(a)

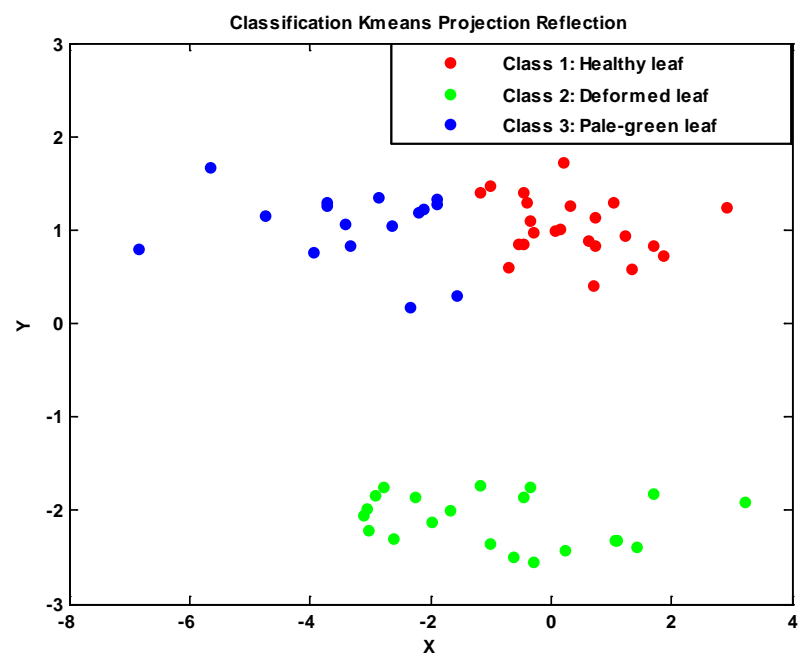

(b)

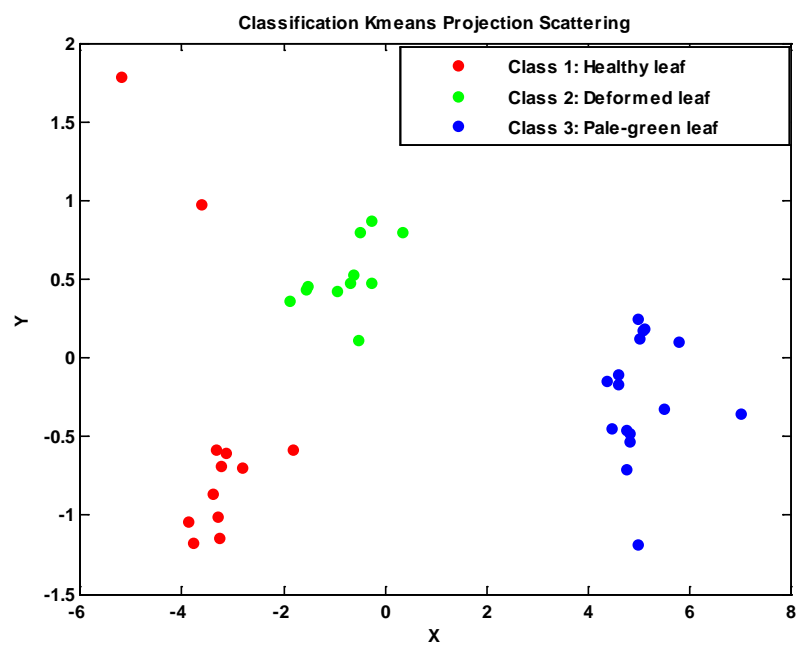

(c)

Figure 6. Projection of infected cassava leaves by the African cassava mosaic virus in transmission (a), reflection (b) and scattering (c) modes, using K-means. 


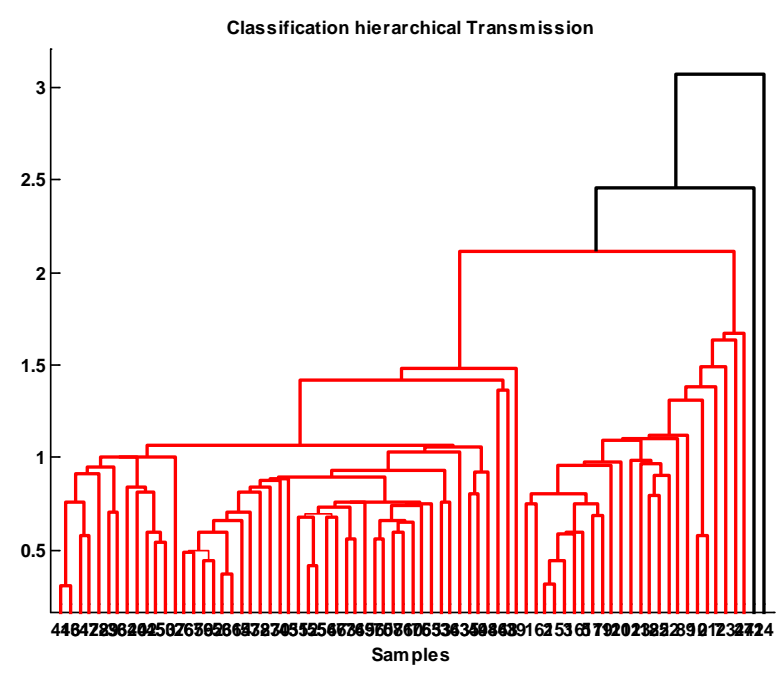

(a)

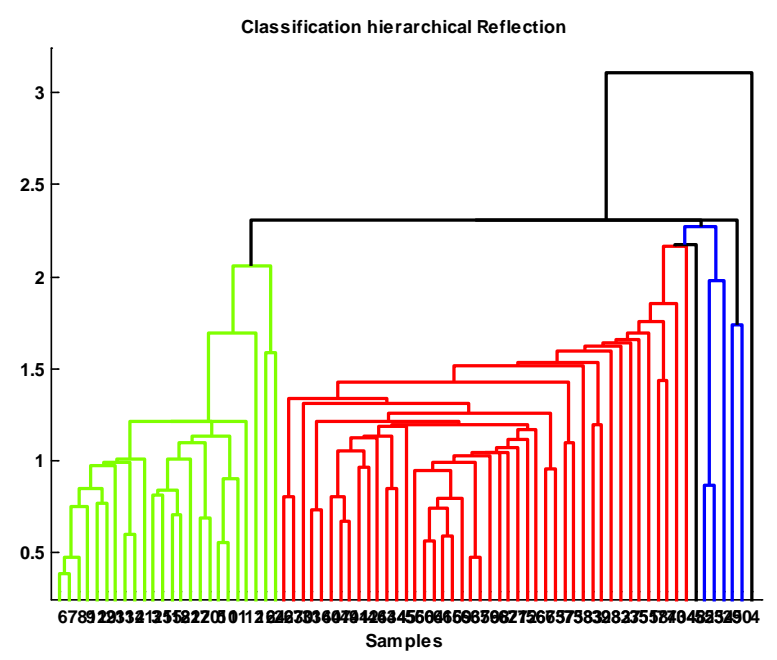

(b)

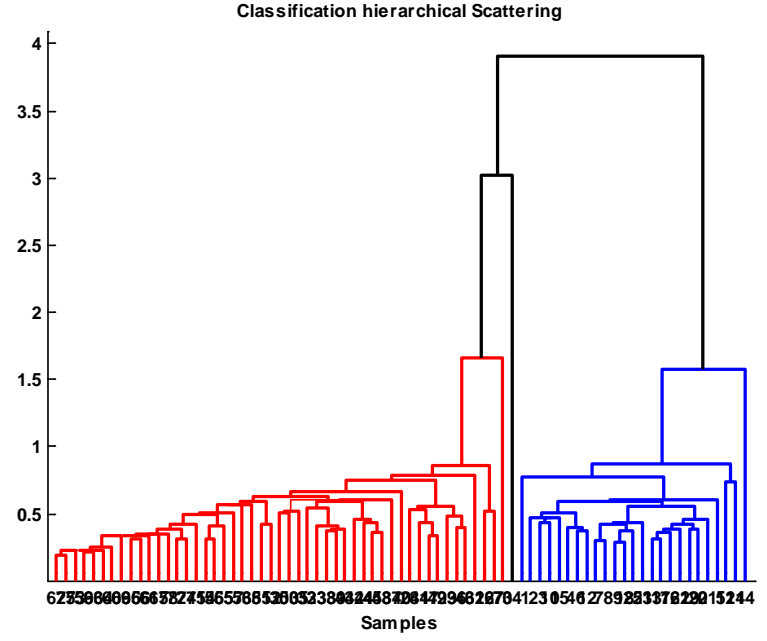

(c)

Figure 7. Projection of stressed cassava leaves by the African cassava mosaic virus in virus in transmission (a), reflection (b) and scattering (c) modes, using AHC. 


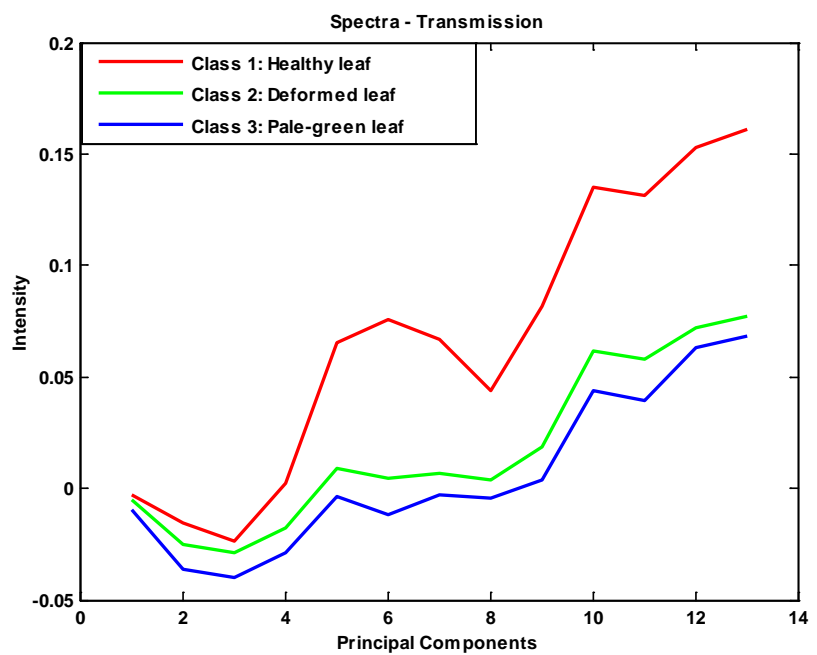

(a)

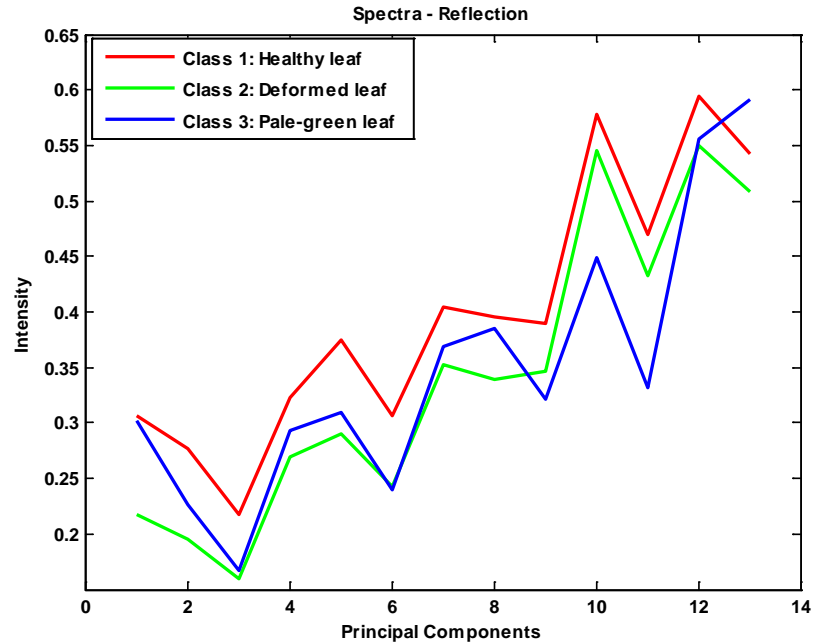

(b)

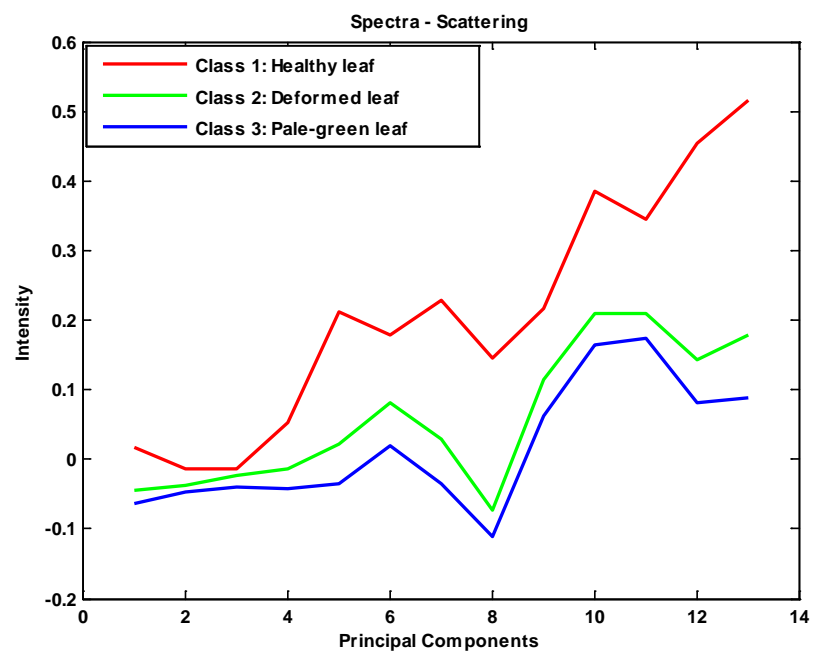

(c)

Figure 8. Spectral representation of the different classes 1, 2 and 3 in transmission (a), reflection (b) and scattering (c) modes. 
of intensities than a clear-green and dark-green leaf (class 3). Generally, when a plant leaf transmits maximum light, we affirm that its chlorophyll concentration rate isn't important [14]. In this case, we can say that this infected leaf (class 2) doesn't normally play the role of photosynthesis. Nevertheless, a clear-green and darkgreen leaf (class 3) absorbs the maximum of wavelength in terms of intensities according to our selected spectral bands characteristics. Its rate of chlorophyll concentration is sufficient to answer the phenomenon of photosynthesis to resist the ACMV. The effect of this infection hasn't influence on the crop. In terms of intensity, we distinguished the spectroscopic behavior of infected leaves in which the characteristics are different. In transmission mode, the stress produced by a clear-green and dark-green leaf (class 3 ) gives a better yield than a stress emitted by a leaf in which the limb is deformed (class 2) by the virus of African cassava mosaic.

\subsubsection{In Reflection Mode}

From visible to near infrared depending on the thirteen wavelengths, the selected samples have similar spectral behavior in reflection mode. In terms of intensities, the healthy cassava leaf (class 1) reflects more light than the leaves infected by ACMV. On the other hand in the ultraviolet, the visible and at $940 \mathrm{~nm}$, the spectral signature of a leaf in which the limb has been deformed by this virus (class 2) reflects less light than a light-green and dark-green leaf (class 3). We observe a contrary effect for the other wavelengths in the near infrared. Some studies have shown that when the reflectance and transmittance are related to the optical properties of plant leaves, a stress can cause degradation of chlorophyll pigments by increasing the reflectance and transmittance in the visible [15]. Their transmittance and reflectance are not related to their biochemical content and their anatomical structure depends on environmental factors and species. Therefore in reflection mode; the stress produced by these infected cassava leaves has an influence on the crop yield. However, the multi-spectral imaging technique applied to our infected samples showed that the reaction of these two infected leaves will not cause a degradation of chlorophyll pigments in the three bands ( $375 \mathrm{~nm}-940 \mathrm{~nm}$ ).

\subsubsection{In Scattering Mode}

The spectral signature of the healthy cassava leaf (class 1), has a higher light scattering variation than the spectra of infected leaves (class 2 and class 3 ) in terms of intensity from ultraviolet to near infrared according to the thirteen wavelengths used. The infected leaves have the same spectral behavior according to the bands used. In terms of intensity, the limb of a deformed leaf (class 2) by the African cassava mosaic diffuses more light than a light-green and dark-green leaf (class 3 ) in the spectral lines from ultra-violet to the near infrared. This strong diffusion may be due to cellular interfaces air-walls [15]. We notice that when a cassava leaf absorbs less chlorophyll, it will diffuse more light. This applies to a leaf infected by ACMV. Thus, we can assert that this phenomenon may be due to the geometric (shape and size), optical (reflection, transmission, absorption, etc.) and physical-chemical properties (temperature, water content, leaf chlorophyll, soil organic matter...) etc.

\section{Conclusion}

After characterizing the leaves infected by the virus of African cassava mosaic, we notice that with the application of techniques and approaches used in this article, our imager is able to differentiate the spectroscopic behavior of cassava leaves infected by this virus in three modes (transmission, reflection and scattering). It stands out from this study that the diseased leaves because of the African cassava mosaic have a production yield which depends on their optical properties. The optical spectral analysis and the formation of multi-spectral images provide powerful means to characterize samples in a large variety of applications. We will continue this study to characterize the evolution of infected leaves by ACMV in Ivory Coast using multi-spectral imaging.

\section{Acknowledgements}

We would like to thank the International Science Programme (ISP) for financing the project. We also thank Prof. S. Svanberg, Dr. M. Brydegaard, Mr. A. Merdasa for their technical support and Prof. T. A AGNEROH for providing the ACMV infected plants leaves.

\section{References}

[1] Boni, N., Zohouri, G.P., Yapi-Gnaoré, V. and Djédji, C. (2008) Bien cultiver le manioc en Côte d’Ivoire. Centre Na- 
tional de Recherche Agronomique, Abidjan, Cote d'Ivoire, 1-4.

[2] Jean, S. (1989) Traité de pathologie végétale. Presses agronomiques de Gembloux, Université de Cornell, Cornell, 621 p.

[3] Denis, F. (1987) Epidémiologie de la mosaïque africaine du manioc en Côte d'Ivoire. Etudes et Thèses, ORSTOM, Paris, $204 \mathrm{p}$.

[4] Ahohuendo, B.C. and Sarkar, S. (1994) Infection de Nicotiana benthamiana et de Manihot esculenta par le virus de la mosaïque africaine du manioc: Aspects ultrastructuraux. Bulletin de la Recherche Agronomique du Bénin, 10, 5-12.

[5] Ambang, Z. (2009) Résistance à la mosaïque virale de Manihot glaziovii par greffage sur Morchella esculenta. Tropicultura, 27, 8-14.

[6] Lefèvre (1989) Recherche des souches de l'espèce sauvage de manioc Manihot glaziovii résistantes aux maladies et aux conditions environnementales. ORSTOM, Abidjan, Cote d'Ivoire, 11-19.

[7] Dubern, J. (1976) Quelques propriétés de la Mosaique Africaine du Manioc. Journal of Phytopathology, 96, 25-39. http://dx.doi.org/10.1111/j.1439-0434.1979.tb01617.x

[8] Zoueu, J.T., Ouattara, S., Touré, A., Safi, S. and Zan, S.T. (2009) Spectroscopic Approach of Multispectral Imaging of Plasmodium Falciparum Infected Human Erythrocytes. IEEE Proceedings of ICTON Mediterranean Winter Conference, 23, 1-7.

[9] Merdasa, A., Brydegaard, M., Svanberg, S. and Zoueu, J.T. (2013) Staining-Free Malaria Diagnostics by Multispectral and Multimodality Light-Emitting-Diode Microscopy. Journal of Biomedical Optics, 18, Article ID: 036002. http://dx.doi.org/10.1117/1.JBO.18.3.036002

[10] Zoueu, J.T. and Zan, S.G.T. (2012) Trophozoite Stage Infected Erythrocyte Contents Analysis by Use of Spectral Imaging LED Microscope. Journal of Microscopy, 245, 90-99.

[11] Besse, P.C. (1992) PCA Stability and Choice of Dimensionality. Statistics \& Probability Letters, 13, 405-410.

[12] Tzeng, D.-Y. and Berns, R.S. (2005) A Review of Principal Component Analysis and Its Applications to Color Technology. Color Research \& Application, 30, 84-98. http://dx.doi.org/10.1002/col.20086

[13] Ringnér, M. (2008) What Is Principal Component Analysis? Nature Biotechnology, 26, 303-304

[14] Avril, B.L. (2007) Mesure et modélisation des propriétés optiques spectrales et directionnelles des feuilles. Ecole Doctorale des Sciences de l'Environnement d'Ile de France. Universite Paris 7-DENIS DIDEROT UFR DE PHYSIQUE.

[15] Krogmann, D. and Govindjee (2004) Discoveries in Oxygenic Photosynthesis (1727-2003): A Perspective. Photosynthesis Research, 80, 15-57. http://dx.doi.org/10.1023/B:PRES.0000030443.63979.e6 